\title{
Learning in honeybees as a function of amount of reward: Rejection of the equal-asymptote assumption
}

\author{
P. A. COUVILLON, YOUNGLIM LEE, and M. E. BITTERMAN \\ University of Hawaii, Honolulu, Hawaii
}

\begin{abstract}
Foraging honeybees were trained individually with successively presented targets differing in odor, one containing $5 \mu \mathrm{l}$ and the other $20 \mu \mathrm{l}$ of a $50 \%$ sucrose solution, after which preferences were measured in choice tests. In Experiment 1, there were either 8 training trials with each tar-

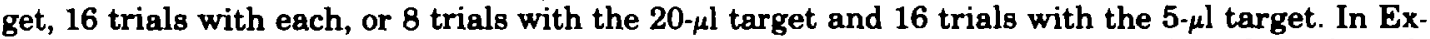
periments 2 and 3, the odor-amount relation was reversed after either 24 or 16 trials with each target. In Experiment 4, differential reward was introduced only after two, four, or six feedings-torepletion on each target. All of the results could be simulated quantitatively and with considerable accuracy on the assumption that the attractiveness of an odor is given by the strength of its association with sucrose; that asymptotic associative strength is an increasing function of amount of reward; and that choice between two odors is determined by their relative associative strength.
\end{abstract}

In recent experiments on appetitive conditioning in honeybees (Buchanan \& Bitterman, 1988), individual foragers were fed on successively presented targets of two different colors, one always containing $5 \mu l$ and the other $20 \mu 1$ of a $50 \%$ sucrose solution, after which preferences were measured in unrewarded choice tests. When the number of training trials with the two colors was the same, the $20-\mu$ l color was preferred, but the difference in amount could be offset by a difference in frequency: When the number of 5- $\mu 1$ training trials was twice the number of $20-\mu 1$ trials, neither color was preferred, and when the number of $5-\mu 1$ training trials was three or more times the number of 20- $\mu 1$ trials, the 5- $\mu 1$ color was preferred.

What may seem at first to be the simplest way of dealing with such results is to assume that colors and amounts are associated-the two associations differing both in their first terms and in their second terms (or significates)and that choice is based on the remembered or "represented" outcomes. The tradeoff between frequency and amount suggests, however, that choice depends not only on what is represented (the substance of each representation), but in some sense also on the strength of representation. A rigorous representational account would require some further assumptions about the growth of associative strength with experience and about the interaction of strength and substance in the determination of choice.

A more parsimonious, nonrepresentational way of dealing with the results is to assume that the two associations

This research was supported by Grant BNS-9010609 from the National Science Foundation. An REU supplement permitted the participation of Julie Nagrampa, to whom the authors are indebted for assistance in collecting the data. Younglim $L e$ is now at Yale University. Requests for reprints should be addressed to the Bekesy Laboratory of Neurobiology, 1993 East-West Road, Honolulu, Hawaii 96822. have a common significate (say, sweet taste) and that choice is based on associative strength, which varies with both frequency and amount of reward. One possibility suggested by the vertebrate literature is that the effect of amount of reward is on the asymptote of the acquisition function (Hull, 1943); another is that the slope of the function is affected as well as the asymptote (Rescorla \& Wagner, 1972). On the basis of the information available to them, Buchanan and Bitterman (1988) were unable, however, to reject the possibility that the effect in honeybees is on the slope of the function alone (the equalasymptote assumption). All of their data could be simulated accurately with the linear equation

$$
\Delta V=\alpha \beta(\lambda-V)
$$

for computing the growth of associative strength on each rewarded trial $(V$, the preexisting strength; $\Delta V$, the increment in strength; $\alpha$, the salience of each color, taken as $1 ; \beta$, the growth rate, taken as .02 for $5 \mu 1$ of sucrose and as .04 for $20 \mu \mathrm{l}$; and $\lambda$, the asymptotic strength, taken as 1 for both amounts of sucrose), together with an exponential equation for computing the probability of choice on the basis of relative associative strength that was borrowed from earlier work on discriminative learning in honeybees (Couvillon \& Bitterman, 1987, 1988).

Nor could the equal-asymptote assumption be rejected on the basis of subsequent experiments that were designed to constrain it more severely (Buchanan \& Bitterman, 1989). In the first of them, one of the two colors contained $5 \mu \mathrm{l}$ of sucrose solution on Trials 1-16 and $20 \mu \mathrm{l}$ of sucrose solution on Trials 17-32, while the opposite was true of the other color. If the number of large-reward trials in such an experiment is the same for each color and if the number of small-reward trials also is the same for each color, the equal-asymptote assumption requires that their 
terminal associative strengths be the same, and a subsequent choice test in fact showed no preference for either color. In a second experiment, there were 12 feedings-torepletion on one of the colors and 12 feedings-to-repletion followed by $155-\mu 1$ trials on the other color. If, as the equal-asymptote assumption implies, $V$ can only increase (however slightly) on a rewarded trial, the added 5- $\mu 1$ trials would not be expected to decrease the attractiveness of the second color, and a choice test in fact showed no preference for either color. Support for the equalasymptote assumption was provided also by a subsequent experiment (Lee \& Bitterman, 1990a), in which a choice test made after prolonged training with 20 - and 5- $\mu$ l colors showed only a small and statistically unreliable preference for the $20-\mu l$ color.

These results differ markedly from the results of analogous experiments with rats and monkeys which often have been interpreted in representational terms. In runway experiments with rats, for example, the effects of training with small reward followed by large reward are clearly distinguished from the effects of training with large reward followed by small reward, decrements in amount of reward producing substantial decrements in performance (Capaldi, 1970; Capaldi \& Lynch, 1967). In choice experiments with rats and monkeys, stimuli paired with large reward are persistently preferred to stimuli paired with small reward (Davenport, 1970; Hill \& Spear, 1963). It would be premature, however, to conclude that learning in honeybees differs in some fundamental way from learning in the other animals, because the range of experiments with honeybees still is relatively narrow and because the only support for the conclusion is provided by the null outcomes of choice tests made at the conclusion of training, which may be misleading. In recent experiments designed to provide more direct information on the course of acquisition, latency of response to the $5-$ and $20-\mu 1$ colors declined in orderly fashion as training continued but did not vary with amount of reward (Lee \& Bitterman, 1990a).

The present experiments were suggested by the possibility that the unusual results obtained in the previous experiments with honeybees might be understood on the assumption that the effective variable in those experiments was delay rather than amount of reward. Trained to choose between two otherwise identical targets of which one contains a drop of sucrose and the other nothing, honeybees locate 5- $\mu 1$ drops much less readily at the outset than they do 20- $\mu 1$ drops, although experience with larger drops improves the detection of smaller drops (Walker, Lee, \& Bitterman, 1990). An association between color and sucrose therefore may develop more rapidly with $20-\mu 1$ drops than with 5- $\mu$ l drops because of closer contiguity between the perception of the color of the target and the initial taste of the sucrose, a common asymptote being approached as the animals learn to detect the small drops more quickly in the course of continued training. A previous effort to equate delay by using small, readily discriminable white dots to mark the locations of the drops on colored targets was unsuccessful because the dots seemed to overshadow the colors (Lee \& Bitterman, $1990 \mathrm{~b})$. When, however, the dotted targets differed in odor rather than color, a clear preference for the $20-\mu \mathrm{l}$ odor developed that could be attributed to amount apart from delay of reward, and in the experiments now to be reported, the role of amount of reward was examined under those conditions. The strategy was to try to replicate with dotted targets differing in odor some of the results previously obtained with undotted targets differing in color.

\section{EXPERIMENT 1}

In this experiment, further evidence of an uncontaminated effect of amount of reward was sought, and the relation between frequency and amount of reward was studied. Three groups of animals were trained, one with $820-\mu 1$ trials with one odor and $85-\mu 1$ trials with the other, a second with $1620-\mu 1$ and $165-\mu 1$ trials, and a third with $820-\mu \mathrm{l}$ and $165-\mu \mathrm{l}$ trials, after which preferences were measured as before in unrewarded choice tests. Previous work with colored targets had shown the difference in amount to be compensated almost exactly by a 2:1 difference in frequency when the total number of trials was relatively small (Buchanan \& Bitterman, 1988).

\section{Method}

Subjects. The subjects were 24 honeybees (Apis mellifera) from our own hives situated near the laboratory. All were experimentally naive.

Procedure. The training situation was like that used by Lee and Bitterman (1990a). It consisted of two immediately adjacent windows (each $55 \mathrm{~cm}$ wide and $55 \mathrm{~cm}$ high) separated by a thin $(2-\mathrm{cm})$ wooden partition around which the animal was required to fly from one window to the other. In the pretraining, experience with both windows was given. A single bee was selected at random from a group of foragers at a feeding station that provided a $10 \%-15 \%$ sucrose solution, carried to the laboratory, and set down at a large drop $(>100 \mu \mathrm{l})$ of $50 \%$ sucrose solution on a pretraining target that was centered on the shelf of one of the two windows (the left for half the animals and the right for the rest). The animal was marked with a spot of colored lacquer as it fed to repletion, after which it was permitted to leave for the hive. Typically, the animal (adapted to $50 \%$ sucrose and now finding the lower, feeder-concentration unacceptable) would come back to the laboratory after a few minutes, continuing to shuttle back and forth from the hive as long as sucrose was available there. If the marked animal did not come back after its first placement, it was carried again from the feeding station, where it usually could be found, to the pretraining target. When the animal did return to the first window, it was picked up after a few seconds of feeding and placed on a pretraining target centered on the shelf of the alternative window, where it was permitted to feed to repletion. On subsequent visits, a pretraining target was presented twice at the second window and once again at the first. The pretraining ended after the animal had returned twice to each window of its own accord.

The targets were covered petri dishes of gray plastic, $5 \mathrm{~cm}$ in diameter. Drilled in the cover of each dish, $6 \mathrm{~mm}$ from its outer circumference, was a circle of 16 equally spaced holes, $3 \mathrm{~mm}$ in diameter, and at its center was a painted white dot, $4 \mathrm{~mm}$ in diameter. The dishes contained pieces of cotton batting that were impregnated with scents. In all, there were three sets of targets, those of one set scented with peppermint, those of the second scented 
with geraniol, and those of the third (used only in the pretraining) scented with both. The covers of the targets used on each visit were washed and exchanged for others in their sets after the visit in order to randomize extraneous stimuli.

Arriving from the hive on each training visit, an animal found either a peppermint or a geraniol target centered on the shelf of one of the windows, the left window on half the visits and the right window on the other half, in quasi-random order. When the animal landed on the target and made contact with the reward, a second target-peppermint or geraniol, in quasi-random order-was centered on the shelf of the adjoining window, to which the animal would fly after taking the sucrose on the first target. Then the first target was removed, another new target was centered on the shelf of the arrival window, and so forth, until the animal was replete and returned of its own accord to the hive. Although the animal itself determined the length of each visit, a running record kept by the experimenter made it possible to control to a considerable extent the number of 20- and 5- $\mu 1$ presentations. For Group 8-8 $(n=8)$, the mean number of trials was exactly 8 with each odor in a mean of 4 visits; for Group 16-16 $(n=8)$, it was 15.9 trials with the 20- $\mu$ l odor and 16 with the 5- $\mu$ l odor in 6.9 visits, and for Group 8-16 $(n=8), 8.1$ trials with the $20-\mu 1$ odor and 16.1 with the $5-\mu l$ odor in 5.5 visits. The $20-\mu l$ odor was peppermint and the 5- $\mu 1$ odor geraniol for half the animals in each group; the opposite was true for the other half.

After the last training visit, each animal returned from the hive to find a pair of fresh targets, one scented with peppermint and the other with geraniol, set $10 \mathrm{~cm}$ apart in a lateral arrangement on the sill of one of the windows, the left window for half the animals in each group and the right window for the rest. For half the animals in each group, the target with the $20-\mu 1$ odor was to the left of the other, and for the remaining animals it was to the right, each target now containing a 10- $\mu 1$ drop of tap water (unacceptable, and distinguishable from the sucrose solution only by taste). Upon encountering water on one of the targets, the animal would leave it, then return to it or go to the other, leave again, return again (often only briefly, with no attempt to drink), and so forth, the interval between successive responses increasing as the test continued. All actual contacts with each target, however brief, during a 10 -min period were recorded by the experimenter, who pressed one of two handheld switches that activated counters programmed to print stored frequencies at $30-\mathrm{sec}$ intervals.

\section{Results}

In Figure 1, the test performance of the three groups is plotted in terms of the mean cumulative number of responses to each odor in successive 30-sec intervals. As the curves show, there was a substantial preference for the 20- $\mu$ l odor in Groups 8-8 and 16-16, but not in Group 8-16. An overall analysis of variance-based in accordance with our standard practice on uncumulated 30-sec scores in 2.5-min blocks-yielded an insignificant group effect $[F(2,21)<1]$, but a significant stimulus (20 vs. $5 \mu 1)$ effect $[F(1,21)=46.37, p<.0001]$, a significant group $\times$ stimulus interaction $[F(2,21)=3.64, p=$ .0438], and a significant group $\times$ stimulus $\times$ block interaction $[F(6,63)=3.85, p=.0025]$. Separate analyses yielded a significant stimulus effect for Group 8-8 $[F(1,7)$ $=70.30, p=.0001]$ and Group $16-16[F(1,7)=15.21$, $p=.0059]$, but not for Group 8-16 $[F(1,7)=2.66$, $p=.1472]$.

The preferences for the $20-\mu 1$ odor shown by Groups 8-8 and 16-16 point to the effectiveness of amount of reward apart from delay of reward on the plausible assump-

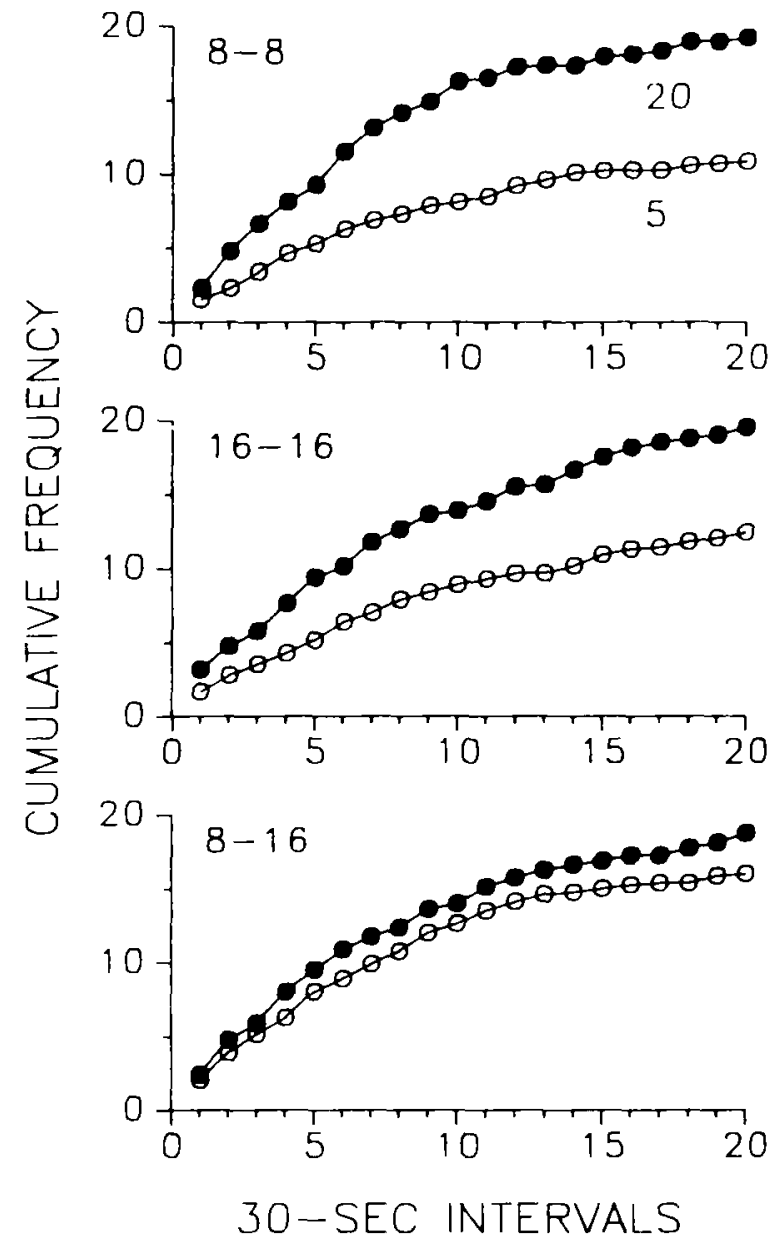

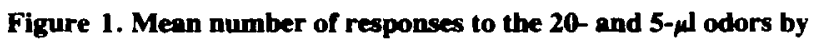
the three groups in the choice test of Experiment 1.

tion that the use of dotted targets rules out the possibility of differential delay. The lack of a preference for the $20-\mu 1$ odor in Group 8-16, for which there were twice as many $5-\mu 1$ as $20-\mu l$ trials, confirms Buchanan and Bitterman's (1988) results for color and gives some reason to anticipate that the color and odor results may not prove to be very different after all. It should be noted, however, that the tradeoff between frequency and amount of reward does not uniquely support the equal-asymptote hypothesis. Where the frequencies are relatively small, a tradeoff may occur even if the two acquisition functions have different asymptotes.

\section{EXPERIMENT 2}

A potentially more instructive test is provided by reversal training. In an experiment to which reference already has been made, Buchanan and Bitterman (1989) gave 16 $20-\mu 1$ trials with one color and $165-\mu 1$ trials with another, followed by 16 additional trials with each color in which the color-amount relationship was reversed. The subse- 


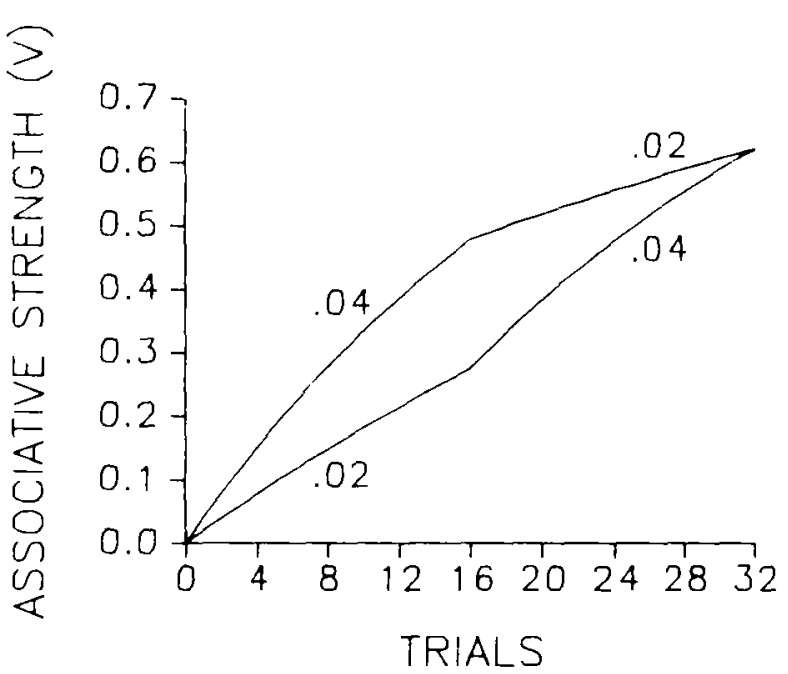

Figure 2. Hypothetical growth of associntive strength in the course of 16 trials with a langer reward followed by 16 trials with a smaller reward, or 16 trials with the smaller reward followed by 16 trials with the larger. $\beta=.02$ for the smaller reward, $\beta=.04$ for the larger reward, and $\lambda=1$.

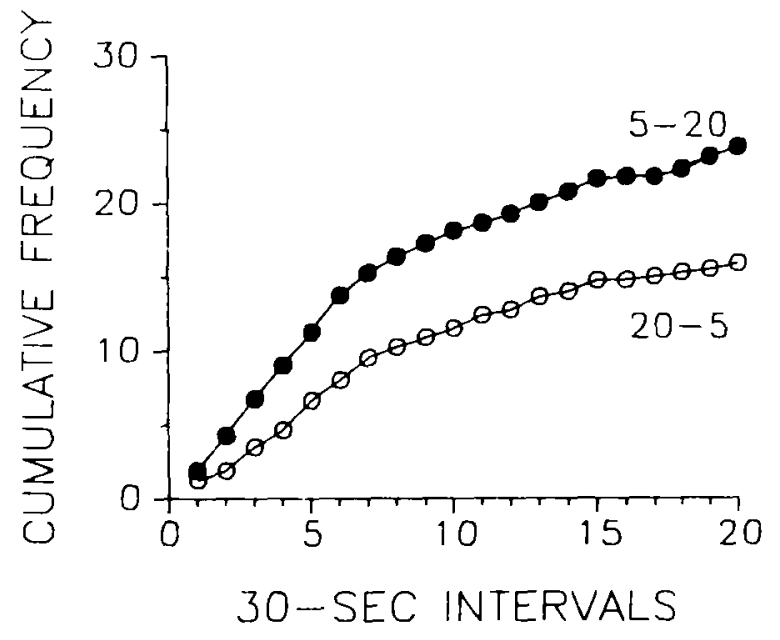

Figure 3. Mean number of responses to the 5-20 and 20-5 aiors in the choice test of Experiment 2.

quent choices of the animals showed no preference for either color, an outcome predicted on the equal-asymptote assumption, as Figure 2 illustrates. If two acquisition functions approach a common asymptote (for convenience, taken as 1) at different rates, it can be shown in general that

$$
V_{m+n}=V_{n+m}=1-\left(1-V_{0}\right)\left(1-\beta_{1}\right)^{m}\left(1-\beta_{2}\right)^{n},
$$

where $\mathbf{V}_{0}$ is the initial associative strength of each color (or odor), $V_{m+n}$ and $V_{n+m}$ are their terminal associative strengths, $m$ and $n$ are the numbers of trials with the two amounts of reward (here, $m=n$ ); and $\beta_{1}$ and $\beta_{2}$ are the learning rates. The argument, however, is one-sided; although a clear preference unequivocally contradicts the equal-asymptote assumption, the lack of a preference does not require it. The present experiment was like Buchanan and Bitterman's except that dotted targets differing in odor rather than undotted targets differing in color were used and the planned number of pre- and postreversal trials with each stimulus was increased from 16 to 24 in order to provide greater opportunity for the development of a postreversal preference.

\section{Method}

Subjects. The subjects were 8 foragers from our own hives, all experimentally naive.

Procedure. The experimental situation and the pretraining method were the same as in Experiment 1 . The general features of the training also were the same, with control of the number of trials in each stage of the training somewhat less successful than usual. In a mean of 12 prereversal visits, the mean number of trials was 26.4 for the 5-20 odor (the odor providing $5 \mu \mathrm{l}$ prereversal and $20 \mu \mathrm{l}$ postreversal) and 26.8 for the 20-5 odor; in the same number of postreversal visits, the mean number of trials was 28.1 for the 5-20 odor and 28.6 for the 20-5 odor. There were, then, about two more postreversal trials than prereversal trials with each odor, although that in itself could not really be expected to produce any measurable preference for the 5-20 odor. The preference test was exactly the same as in Experiment 1, with windows, odors, and target positions balanced over animals as before.

\section{Results}

In Figure 3, performance in the test is plotted in terms of the mean cumulative number of responses to 5-20 and 20-5 odors in successive $30-\mathrm{sec}$ intervals. The curves show a strong preference for the 5-20 odor. Analysis of variance yielded both a significant stimulus effect $[F(1,7)$ $=25.37, p=.0015]$ and a significant stimulus $\times(2.5-$ min) block interaction $[F(3,21)=4.28, p=.0166]$. The equal-asymptote assumption is contradicted.

\section{EXPERIMENT 3}

Experiment 2, which showed a strong postreversal preference for the 5-20 alternative, differed in several procedural respects from Buchanan and Bitterman's (1989) reversal experiment, which showed no preference: A double-window rather than a single-window training situation was used, the stimuli to be discriminated were odors rather than colors, and the scheduled number of training trials with each stimulus in each stage was considerably larger ( 24 rather than 16$)$. Of the three variables, the one of most immediate interest is number of training trials, which clearly is critical for the demonstration of asymptotic inequality; with all other things equal, the probability of a measurable preference, either pre- or postreversal, is small when the number of training trials is small. The procedure in the present experiment was like that in Experiment 2, with the exception that only 16 pre- and postreversal training trials were scheduled.

\section{Method}

Subjects. The subjects were 9 foragers from our own hives. (The design called for 8 subjects, but a 9th was added inadvertently.) All the animals were experimentally naive. 


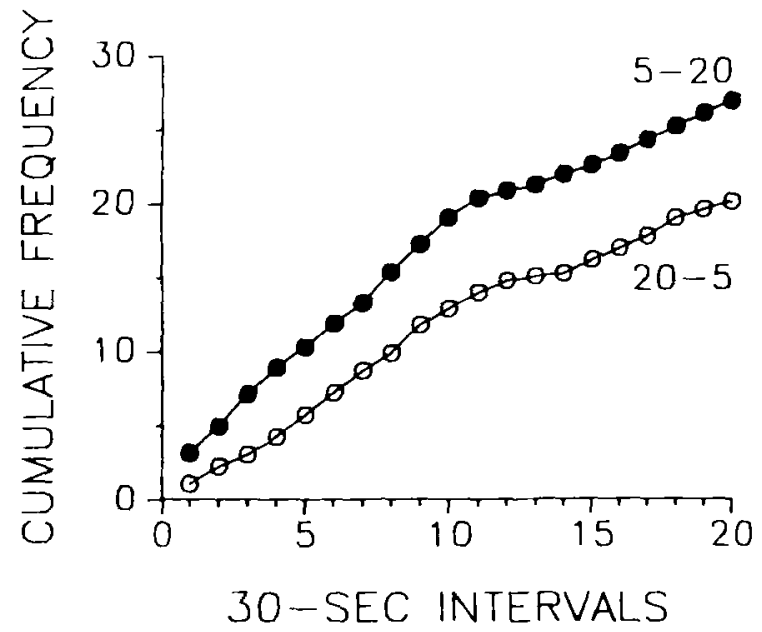

Figure 4. Mean number of responses to the 5-20 and 20-5 odors in the choke test of Experiment 3.

Procedure. The experimental situation, the pretraining procedure, the training procedure, and the subsequent testing procedure were the same as in Experiment 2 . The only difference was the number of training trials and visits. In a mean of 8.4 prereversal visits, the mean number of trials was 16.1 for the $5-20$ odor and 16.2 for the 20-5 odor; in a mean of 8.2 postreversal visits, the mean number of trials was 16.2 for the 5-20 odor and 15.9 for the 20-5 odor.

\section{Results}

In Figure 4, the test performance is plotted in terms of the mean cumulative number of responses to each odor in successive 30-sec intervals. Again, the curves show a preference for the 5-20 odor, although a bit smaller perhaps than in Experiment 2. Analysis of variance yielded both a significant stimulus effect $[F(1,8)=8.04$, $p=.0220]$ and a significant stimulus $\times(2.5-\mathrm{min})$ block interaction $[F(3,24)=4.46, p=.0126]$. Again, the equal-asymptote assumption is contradicted.

\section{EXPERIMENT 4}

The design of this experiment also was suggested by the work of Buchanan and Bitterman (1989), who differentially rewarded two colors after a series of feedingsto-repletion calculated to bring their associative strengths to asymptote. Buchanan and Bitterman's purpose was to test the equal-asymptote assumption, which now can be rejected on the basis of the results of Experiments 2 and 3 . In the present experiment, the number of feedings-torepletion given before the differential training was varied over groups. It was anticipated that the results, taken together with those of Experiments 1-3, would permit some reasonable estimates of the different asymptotes supported by the $5-$ and $20-\mu 1$ rewards as well as the rate of learning assumed on grounds of parsimony to be the same for all amounts.

\section{Method}

Subjects. The subjects were 24 foragers from our own hives, all experimentally naive.
Procedure. The experimental situation and the pretraining procedure were the same as in the previous experiments. In the first stage of training, there was a series of visits on each of which the animal found a single target containing a large ( $>100 \mu$ ) drop of sucrose solution from which it fed to repletion. For Group $2 F(n=8)$, there were two such visits to a target scented with one of the odors and two to a target scented with the other; for Group 4F $(n=8)$, there were four visits to each of the odors, and for Group 6F $(n=8)$, six to each of the odors. Each odor was presented equally ofien in each of the two windows, and the sequences of odors and win dows were balanced over groups. In the second stage of training all groups were scheduled to have 16 differentially rewarded trials with each odor, in exactly the same manner as did Group 16-16 of Experiment 1. For Group 2F, the actual means were 16 trials with the $20-\mu$ lodor and 15.3 with the $5-\mu$ l odor in 9.4 visits; for Group 4F, the corresponding means were $15.1,15.4$, and 8.1, and for Group 6F, 16, 15.8, and 8.8. After the training, there was the standard preference test.

\section{Results}

In Figure 5, the test performance of each of the three groups is plotted in terms of the mean cumulative number of responses to each odor in successive 30-sec intervals. As the curves show, there was a preference for the $20-\mu 1$ odor in all groups, somewhat larger in Group $2 \mathrm{~F}$

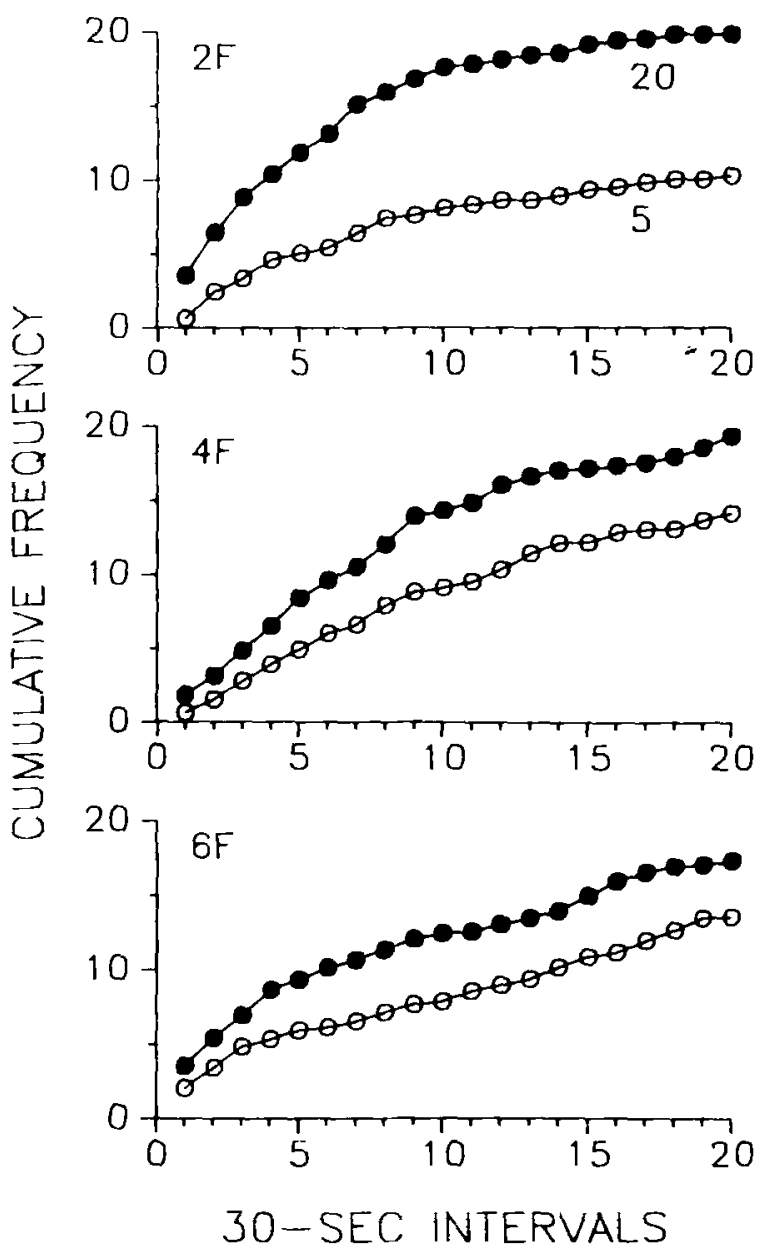

Figure 5. Mean number of responses to the 20- and 5- $\mu$ odors by the three groups in the choice test of Experiment 4. 
than in the others. An overall analysis of variance yielded a significant stimulus $(20$ vs. $5 \mu 1)$ effect $[F(1,21)=$ $27.23, p<.0001]$ and a significant stimulus $\times(2.5-\mathrm{min})$ block interaction $[F(3,63)=20.29, p<.0001]$; it did not yield a significant group effect $[F(2,21)<1]$, a significant group $\times$ stimulus interaction $[F(2,21)=2.18$, $p=.1378]$, or a significant group $\times$ stimulus $\times$ block interaction $[F(6,63)=1.04, p=.4041]$.

\section{DISCUSSION}

The results of Experiments 2 and 3, which directly contradict the equal-asymptote assumption, leave us still with the problem of whether preferences can be explained in terms of associative strength or whether representation is required. The simplest alternative to the equal-asymptote assumption is the nonrepresentational unequal-asymptote assumption - the assumption that the effect of amount of reward is on the asymptote rather than the slope of the acquisition function-and it seems reasonable to ask first whether the results of the entire set of experiments can be understood on that assumption.

To answer the question, we turned to a general (continuity) theory of discriminative learning in honeybees based on the data of a wide range of discrete-trial choice experiments with one trial per visit and feeding-torepletion as reward (Couvillon \& Bitterman, 1987, 1988, 1991). Continuing to assume, as in the earlier work, that feeding-to-repletion supports the development of maximal associative strength-that the asymptote $(\lambda)$ of the acquisition function for the largest possible reward is 1-we looked for values of $\lambda$ for the 20- and 5- $\mu 1$ rewards that would permit accurate simulation of the present data, and we were able to find them. The best fit, with $\lambda=.9$ for $20 \mu \mathrm{l}$ and .7 for $5 \mu \mathrm{l}$ (root-mean-square deviation $=.018$ ), is shown in Figure 6, which compares the simulated and observed proportions of response to the $20-\mu \mathrm{l}$ odor in $\mathrm{Ex}$ periments 1 and 4 and to the 5-20 odor in Experiments 2 and 3. The three sets of points connected to facilitate comparison are those for Groups 8-8, 16-16, and 8-16 of Experiment 1, for the reversal groups of Experiments 2 and 3 (labeled R24 and R16, respectively), and for Groups $2 \mathrm{~F}, 4 \mathrm{~F}$, and $6 \mathrm{~F}$ of Experiment 4 . It should be noted that discrepancies between simulated and observed values are attributable not to theoretical error alone, but also to random error in the data, which means (given the small number of animals in each group) that the theoretical error is even smaller than may appear.

It should be noted that the parametric values used in this simulation were exactly those which provided the best fit to all of the earlier, discrete-trial choice data, with two exceptions. One difference was in the choice functionthe function relating probability of choice to relative associative strength used in the earlier experiments to compute the proportion of animals choosing the rewarded alternative on each training trial and here to compute the proportion of responses to the preferred alternative in the 10-min unrewarded test that followed the training. The best fits to the earlier data were given by a cluster of five similar choice functions (described by two parameters, $K$ and $s$, reflecting their curvature and steepness). The one used here $(K=.5, s=.5)$ belonged to this cluster, but it was not the one that gave the very best fit to the earlier data. The latter $(K=.75, s=.625)$ also gave a good fit to the present data, though not the very best one.

Before it can be determined which choice function gives the best fit to both sets of data combined, a second parametric discrepancy must be resolved. Simulations of the earlier data suggested that the down $\beta(\mathrm{D} \beta)$-the rate at which $V$ declines when on any trial it exceeds the $\lambda$ for the outcome of response on that trial-was rather large (on the order at least of .2), but simulations of the present data suggested that it was much smaller; the fit illustrated in Figure 6 is based on $D \beta=.03$. One reason for the discrepancy may be that, in the simulations of the earlier data, the $V$ of an unrewarded alternative was decremented only once on any trial, although often a real animal would make a series of unrewarded responses before shifting to the rewarded alternative; that is, no account was taken of repetitive errors, which would tend to inflate the estimate of $\mathrm{D} \beta$. In the present experiments, by contrast, there was only one response on each training trial. It might be instructive to record and try to simulate repetitive errors in replications of some of the earlier experiments. Another reason for suspecting that $D \beta$ might have been overestimated in the earlier experiments is that no distinction was made between the reduction of $V$ per se and the possibility of competing frustration responses generated by relatively large differences between $V$ and $\lambda$ (the nonrepresentational equivalent of "unrealized expectation"). With feeding-to-repletion as reward, not only did $V$ tend to be

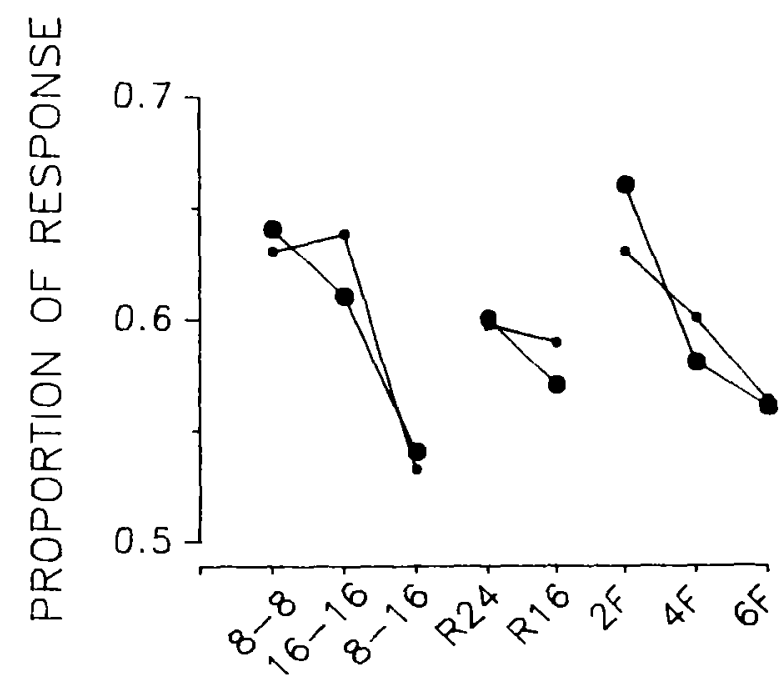

Figure 6. Simulated (small circles) and observed (large circles) proportions of response to the 20- $\mu$ odor in Groups 8-8, 16-16, 8-16, 2F, 4F, and 6F of Experiments 1 and 4 and to the 5-20 odor in Groups R24 and R16 of Experiments 2 and 3. As explained in the text, the three sets of points are connected to facilitate comparison. 
somewhat larger, but the $\lambda$ approached on unrewarded trials was zero; in the present experiments, the greatest possible difference between $V$ and $\lambda$ on any trial was the difference between the $\lambda s$ for the 20 - and $5-\mu$ l rewards. That frustration may have played some role in the earlier experiments is suggested by the overlearning-extinction effect in honeybees (Couvillon \& Bitterman, 1984), which does not in itself require a representational interpretation (Couvillon \& Bitterman, 1991).

Whatever the relation between the two sets of data and the possibility of deriving them from a single theory, it seems clear that the present results do not require us to go beyond the parsimonious, nonrepresentational assumption that the effect of amount of reward is on the asymptote of the acquisition function. The possibility that the slope of the function is affected as well is not, of course, ruled out, although there is as yet no need of that assumption. Nor is the possibility ruled out that amount of reward is represented as such, having no effect at all on the strength of association. What is ruled out by the present experiments is the nonrepresentational equalasymptote assumption, which could not be rejected on the basis of a long series of previous experiments that seemed to set honeybees apart from other animals studied, and it is interesting to ask why the two sets of results should be so different.

One reason may be that the two-window procedure employed here is more sensitive than the single-window procedure which yielded null results in the experiments of Buchanan and Bitterman (1989), because, in alternating between the windows, the animals approach each target in a fairly standard manner. A more important reason may be that odors were used in the present experiments and colors in the earlier ones. If the associative strength acquired by a stimulus paired with sucrose depends on the duration of concurrent exposure, and if odors, but not colors, are processed after landing, it may be that the results for undotted targets differing in color are to be understood as delay effects and only the present results for dotted targets differing in odor are to be understood as pure amount effects. Opfinger $(1931,1949)$ found no evidence of learning after landing, about either color or odor, although other experiments with stationary, as distinct from flying, foragers have pointed to learning about odor but not about color (Walker, Baird, \& Bitterman, 1989). The study of this question has not progressed very far.

\section{REFERENCES}

Buchanan, G. M. . Bitterman, M. E. (1988). Leaming in honey bees as a function of amount and frequency of reward. Animal Leaming \& Behavior, 16, 247-255.

Buchanan, G. M., Bittreman, M. E. (1989). Leaming in honeybees as a function of amount of reward: Tests of the equal-asymptote assumption. Animal Leaming \& Behavior, 17, 475-480.

CAPALDI. E. D. (1970). Effect of initial reinforcer magnitude on subsequent resistance to extinction. Journal of Experimental Psychology, 86, 283-287

CAPALDi, E. J., \& LYNCH, D. (1967). Repeated shifts in reward magniaude: Evidence in favor of an associational and absolute (noncontexuual) interpretation. Jourmal of Experimental Psychology, 75, 226-235.

Couvillon, P. A., Bitterman, M. E. (1984). The overlearning extinction effect and successive negative contrast in honeybees. Joumal of Comparative Psychology, 98, 100-109.

Couvillon, P. A., Bitterman, M. E. (1987). Discrimination of color-odor compounds by honeybees: Tests of a continuity model. Animal Leaming \& Behavior, 15, 218-227.

Couvillon, P. A., a Brttereman, M. E. (1988). Compound-component and conditional discrimination of colors and odors by honeybees: Further tests of a continuity model. Animal Leaming \& Behavior, 16, 67-74.

Couvillon, P. A., Bitterman, M. E. (1991). How honeybees make choices. In J. L. Goodman \& R. C. Fischer (Eds.), The behaviour and physiology of bees (pp. 116-130). Wallingford, U.K.: CAB International

DA VENPORT, J. W. (1970). Species generality of within-subjects reward magnitude effects. Canadian Joumal of Psychology, 24, 1-7.

HiLl, W. F., \& SpeAR, N. E. (1963). Choice between magnindes of reward in a T maze. Journal of Comparative \& Physiological Psychology, 56, 723-726.

Hull, C. L. (1943). Principles of behavior. New York: AppletonCentury-Crofts

LeE, Y., Bitrerman, M. E. (1990a). Leaming in honeybees (Apis mellifera) as a function of amount of reward: Acquisition measures. Joumal of Comparative Psychology, 104, 152-158.

LEE, Y., BitTeRMAN, M. E. (1990b). Learning in honeybees as a function of amount of reward: Control of delay. Animal Leaming \& Behavior, 18, 377-386.

OPfinger, E. (1931). Über die Orientierung der Biene an der Futterquelle. Zeitschrifi fur Vergleichende Physiologie, 15, 431-487.

Opfinger, E. (1949). Zur Psychologie der Duftdressuren bei Bienen. Zeitschrifi fur Vergleichende Physiologie, 31, 441-453.

Rescorla, R. A., Wagner, A. R. (1972). A theory of Pavlovian conditioning: Variations in the effectiveness of reinforcement and nonreinforcement. In A. H. Black \& W. F. Prokasy (Eds.), Classical conditioning II: Current research and theory (pp. 64-99). New York: Appleton-Century-Crofts.

Walker, M. M., Baird, D. L., Bitterman, M. E. (1989). Failure of stationary but not of flying honeybees (Apis mellifera) to respond to magnetic field stimuli. Journal of Comparative Psychology, 103, 62-69.

WalKer, M. M., Lee, Y., Bitterman, M. E. (1990). Transfer along a continuum in the discriminative learning of honeybees (Apis mellifera). Joumal of Comparative Psychology, 104, 66-70. 